\title{
Modelo de identificação do impacto futuro de chuvas extremas nos atrasos/cancelamentos de voos
}

\author{
Thiago Adriano dos Santos ${ }^{1}$, Iria Vendrame ${ }^{2}$, Cláudio Jorge Pinto Alves ${ }^{3}$, Mauro Caetano ${ }^{4}$, \\ João Paulo Souza Silva ${ }^{5}$
}

1Instituto Tecnológico de Aeronáutica (ITA), 79thiagoad@ita.br

2Instituto Tecnológico de Aeronáutica (ITA), hiria@ita.br

3Instituto Tecnológico de Aeronáutica (ITA), claudioj@ita.br

4Instituto Tecnológico de Aeronáutica (ITA), caetano@ita.br

${ }^{5}$ Universidade Federal de Goiás, profjpss@gmail.com

\section{Recebido:}

15 de maio de 2017

Aceito para publicação:

30 de fevereiro de 2018

Publicado:

31 de agosto de 2018

Editor de área:

Li Weigang

\section{Palavras-chaves:}

Aeroportos,

Correlação de Spearman

Mudanças climáticas.

\section{Keywords:}

Airport,

Spearman Correlation,

Climate Changes.

DOI:10.14295/transportes.v26i2.1379

\begin{abstract}
RESUMO
Problemas associados aos atrasos e cancelamentos de voos impactam significativamente o desenvolvimento do setor de aviação. Condições climáticas desfavoráveis estão entre algumas das principais razões para esses problemas, o que demanda a análise da interferência do clima nas operações aeroviárias. Nesse sentido, o presente estudo, tomando como referência o caso do Aeroporto Internacional de São Paulo/Guarulhos (GRU), correlaciona eventos de atrasos e cancelamentos de voos aos índices pluviométricos acumulados. Os dados pluviométricos considerados para a estação meteorológica de GRU são referentes aos anos de 2011 a 2015, enquanto as previsões são realizadas para o período entre 2016 e 2020 com o propósito de testar o modelo proposto. Utiliza-se a correlação de Spearman para analisar a relação entre índice pluviométrico e atrasos/cancelamentos de voos para criar um intervalo de valores de chuvas diárias acumuladas que geram esses atrasos/cancelamentos. A correção de Bias, pelo método da transformação de potência, também foi adotada para os dados futuros já corrigidos. Os resultados demonstram que, de acordo com os dados utilizados, haverá um aumento de aproximadamente $15 \%$ na quantidade de atrasos/cancelamentos de voos nos cinco anos considerados no estudo. Além disso, considera-se que haverá um aumento no número de dias de ocorrência desses eventos.
\end{abstract}

\begin{abstract}
Problems associated with flight delays and cancellations significantly affect the development of the aviation industry. Unfavourable weather conditions are among some of the main reasons for these problems, and the mapping and climate predictions are essential for making decisions regarding climate interference in air operations. In this sense, the present study takes as reference the case of São Paulo/Guarulhos International Airport (GRU) and correlates events of flight delays and cancellations to accumulated rainfall indexes. The pluviometric data for the meteorological station of GRU considered refer to the years 2011 to 2015, while the forecasts are made for the period between 2016 and 2020 in order to test the proposed model. Spearman correlation is used to analyse the relationship between rainfall index and flight delays/cancellations to create a range of accumulated daily rainfall values that generate these delays/cancellations. The Bias correction, by the power transformation method, was also adopted for future corrected data. The results show that, according to the data used, there will be an increase of approximately $15 \%$ in the amount of flight delays/cancellations over the five years considered in the study. In addition, it is considered that there will be an increase in the number of days of occurrence of these events.
\end{abstract}

\section{INTRODUÇÃO}

Uma rede de transporte aéreo consiste na união entre aeroportos que podem ser entendidos como os nós da rede. Assim, as operações e voos regulares consistem nas ligações e ramificações 
dessa rede, como um alongamento físico e interligado entre esses aeroportos (JANIC, 2015). Quando algo não esperado acontece com algum desses elementos nessas ligações, a rede toda pode ser prejudicada. Algumas variáveis aparecem como fonte causadora dessas interrupções e podem gerar problemas que muitas vezes não são possíveis de serem sanados de imediato, gerando efeitos prejudiciais em cadeia.

Casos como o de atrasos e cancelamentos de voos apresentam-se como alguns dos principais problemas associados à interrupção das operações dessa rede. Algumas das principais causas desses problemas tornam-se complexas e difíceis de serem mensuradas e gerenciadas, como eventos de situações meteorológicas extremas. Entre esses eventos, índices pluviométricos oscilantes podem ser considerados como de alto impacto nas operações de transporte aéreo.

Estudos sobre eventos meteorológicos impactantes na aviação, como incidências de tempestades formadoras de rajadas de vento, podem ser identificados em Metchko e Monteiro (2014), bem como estudos sobre ocorrência de cancelamentos de voos devido à chuva extrema, como em Koetse e Rietveld (2009). Além desses, há também estudos que buscam mensurar os impactos dos atrasos dos voos nos custos e na dinâmica do transporte aéreo, como em Bendinelli, Bettini e Oliveira (2016), que consideram em suas análises, entre outras, variáveis relacionadas à proporção de atrasos de voos devido ao mau tempo. Já em Santos e Robin (2010), em que se analisam as principais causas para os atrasos de voos em aeroportos europeus, os autores demonstram que a concentração de mercado em determinados aeroportos e companhias aéreas estão entre as principais causas para os atrasos. Sendo assim, nota-se que tais estudos não consideram as situações climáticas em acontecimento, mais incisivamente o clima futuro e como esse provocará alterações nas operações dos aeroportos. Trata-se, portanto, de uma lista de estudos que analisam o passado, mas não fazem projeções futuras.

Sendo assim, o presente estudo aborda esses eventos meteorológicos pluviométricos correlacionando-os aos atrasos e cancelamento de voos, de modo que se possa criar um modelo que mensure cada intervalo de precipitação e os relacione com a quantidade de voos atrasados e cancelados em um determinado intervalo de tempo. Os resultados desse estudo podem proporcionar o desenvolvimento de metodologias para a análise desse problema em aeroportos que operem tanto a partir de regras visuais de voo (VFR) quanto por regras de voo por instrumentos (IFR).

\section{O CLIMA COMO FATOR CRUCIAL PARA CAUSAS E CONSEQUÊNCIAS NO ATRASO E CANCELAMENTO DE VOOS}

Cancelamentos de voos podem estar associados ao seu elevado tempo de atraso para decolagem. Também os atrasos oriundos de desembarque podem conduzir aos atrasos de embarque quando a infraestrutura do aeroporto possui dependência entre esses fluxos. Para que seja decretado o cancelamento de determinado voo, Xiong e Hansen (2013) citam que os tomadores de decisão nessas operações, como os controladores de voo, levam em consideração o tamanho das companhias, valores das tarifas aéreas, quantidade de passageiros e cargas e distância entre os pontos de origem e destino dos voos.

Janic (2015) cita algumas causas que afetam a rede de transporte aéreo, dentre elas destacam-se as ações de infraestrutura e manutenção, grande fluxo aéreo e terrestre, acidentes terrestres, desastres naturais (terremotos, vulcões, maremotos) e, principalmente, eventos climáticos ou mau tempo (denso nevoeiro, chuva intensa e/ou queda de neve, furacões, tornados, etc.). Dependendo do tipo, intensidade e duração desses eventos climáticos, o seu impacto pode 
durar desde algumas horas a vários dias. Alguns desses fatores podem, além de levar ao atraso ou cancelamento de voos, também serem responsáveis por encerramento das operações nesse período. Segundo Benedetto (2002), a segurança de uma aeronave durante o pouso e decolagem está diretamente relacionada às condições da pista e essas, por sua vez, relacionadas ao clima, especialmente à intensidade de chuvas.

Silva (2008) comenta que, durante muitos anos, acidentes aeronáuticos eram tratados como resultado único de falhas do avião (equipamento) ou do piloto, mas com o passar do tempo, esse conceito foi modificado devido a um melhor entendimento das falhas inerentes à aviação. Sabe-se que um acidente é resultado de inúmeras causas, também conhecidas como fatores contribuintes, que juntos desencadeiam uma série de eventos que acabam por ocasioná-lo. Os acidentes e incidentes aéreos envolvem características específicas em cada caso. Entretanto, podem-se distinguir quatro fatores frequentemente relacionados a este tipo de ocorrência, como o fator humano, meteorológico, aeronave e infraestrutura.

Outra abordagem apresentada por Janic (2015) faz uma análise histórica mostrando que 29\% dos acidentes ocorridos nos EUA durante o período de 2001 e 2010 tiveram como responsável o clima. Ocorrências de mau tempo referem-se a redução de $22 \%$ a $48 \%$ na capacidade do sistema de pista nos EUA e de 30\% a 40\% na Europa. Isso demonstra que alterações climáticas, como por exemplo, eventos de precipitações extremas, afetam incisivamente as operações e rotinas de redes de transporte aéreo, resultando em grandes atrasos, e esses podendo levar até ao cancelamento de voos.

Já Koetse e Rietveld (2009) demonstram que eventos climáticos, como ventos fortes e chuvas extremas, são responsáveis por impactos significativos na segurança do transporte aéreo. Um exemplo citado pelos autores demonstra que no Aeroporto Internacional de São Francisco (SFO), nos Estados Unidos, nos meses de verão há grande incidência de tempestades de chuva e essas levam a atrasos significativos e numerosos cancelamentos. Em comparação às situações de bom tempo, os cancelamentos sofrem um aumento por um fator de 2 a 3 vezes quando o mau tempo é somente na parte da manhã, e por um fator de 3 a 4 vezes quando o mau tempo se dá durante todo o dia.

Os eventos extremos de clima, como fortes intensidades de chuva, tem se agravado devido às situações climáticas e suas intensas alterações (Koetse e Rietveld, 2009). As situações climáticas estão associadas à vulnerabilidade e essa refere-se à maneira como um sistema reage às degradações impostas a partir da sua exposição a vários riscos, conforme demonstram Halkos e Tsilica (2014).

Silva (2008) demonstra que o fator meteorológico é o único fator contribuinte para ocorrência de acidente aeronáutico sobre o qual o homem não exerce nenhum controle. As constantes variações e às vezes a violência fazem com que o homem tenha certa dificuldade em lidar com condições meteorológicas. 0 piloto necessita de informações sobre as reais condições de tempo que irá encontrar quando estiver efetuando uma aproximação para pouso. A falta de precisão faz com que muitas vezes o piloto encontre condições adversas com a qual não contava e, consequentemente, para a qual não estava preparado e dependendo das circunstâncias, um acidente pode ser algo inevitável.

Apesar de situações climáticas e seus impactos no transporte aéreo apresentarem-se como questões fortemente debatidas na medida em que essas alterações climáticas podem representar sérias limitações para o setor, nota-se na literatura a existência de lacunas referentes aos atrasos e cancelamentos de voos no futuro levando em consideração os cenários futuros das 
situações climáticas. Algo que correlacione e modele essa problemática com futuros eventos extremos de chuva, bem como cálculos sobre danos futuros associados a essas alterações e os julgamentos sobre os meios de mitigação e adaptações a serem feitas apresentam-se de modo distinto e carente na literatura.

Este estudo relaciona as situações climáticas aos cenários futuros, considerando 4 distintos cenários: A1, A2, B1 e B2, cujas nomenclaturas são apresentadas por Walters e Sebens (2016) e que levam em consideração as emissões de $\mathrm{CO}_{2}$, aumento populacional e aumento do consumo. Cada um desses cenários projeta um futuro climático, em que podem ocorrer eventos de clima extremo, como, por exemplo, altas temperaturas e chuvas intensas. Entretanto, mesmo no estudo apresentado pelos autores, não há uma métrica ou modelo aplicável ao caso de chuvas extremas, sendo o preenchimento dessa lacuna a principal contribuição do presente estudo.

\section{MATERIAIS E MÉTODOS}

\subsection{Dados de atrasos e cancelamentos de voos}

Para a coleta de dados sobre atrasos e cancelamento de voos, foi utilizada uma série histórica de cinco anos (2011-2015), enfatizando quais dessas ocorrências foram provocadas por ações meteorológicas, mais especificamente chuvas intensas. Trata-se de dados públicos obtidos através da Agencia Nacional de Aviação Civil (ANAC) e através de empresas que operam no Aeroporto Internacional de São Paulo/Guarulhos (GRU). Tais dados foram tabulados e organizados diariamente, evidenciando a quantidade de operações atrasadas ou canceladas nesse período. Ressalta-se que futuras alterações nos fluxos de passageiros, bem como possíveis futuros aumentos ou reduções nas operações do aeroporto em estudo não foram consideradas nesse estudo.

\subsection{Dados climatológicos de observação e dados de simulação}

Os dados, especificamente os de precipitação diária, foram obtidos através do banco de dados do Instituto Nacional de Meteorologia (INMET) através de cadastro individual ou obtidos no site do Sistema Integrado de Gerenciamento de Recursos Hídricos (SIGRH). Esses dados são necessariamente referentes ao mesmo período da série histórica dos dados de cancelamento e atrasos de voos. Além de servir para confrontar com os dados de cancelamento e atraso de voos, os dados climatológicos serviram para realizar a correção dos dados de simulação futura na validação do modelo.

Simulações foram realizadas a partir de dados obtidos através de um banco de dados específico fornecido pelo Instituto Nacional de Pesquisas Espaciais (INPE), extraído através do software OpenGrads por meio de linguagem de programação. Esses dados foram calculados e compilados através de um supercomputador do INPE e disponibilizados na linguagem LINUX. Já os dados de precipitação, projetados para o cenário futuro A1B, foram obtidos através do modelo regional ETA, nome derivado da letra grega que indica a coordenada vertical. Uma das características desse modelo consiste na sua precisão de $20 \mathrm{~km}$, no qual as simulações de clima se concentram numa grade de $20 \mathrm{~km}$ por $20 \mathrm{~km}$ de extensão (Santos, 2014).

\subsection{Correlação de Spearman}

Trata-se de uma medida de correlação não-paramétrica, sendo que essa avalia a descrição da relação entre duas variáveis, sem fazer suposições sobre a distribuição de frequências dessas variáveis. Como não requer a suposição de que a relação entre as variáveis é linear, essa se difere 
do coeficiente de correlação de Pearson, por exemplo, e pode ser utilizada para as variáveis medidas no nível ordinal (Alves et al., 2015). Essa medida comumente tem sido indicada quando determinada variável apresenta pontos distantes dos demais ou quando o gráfico de dispersão sugere uma relação crescente ou decrescente na curva considerando X e Y. Como comparação utilizada entre a variável climática chuva e a incidência de atrasos/cancelamentos de voos apresenta tais características, optou-se por utilizar essa medida, cujo coeficiente de correlação pode ser obtido a partir da Equação 1.

$$
\rho=1-\frac{6 \sum d^{2}}{n\left(n^{2}-1\right)}
$$

Sendo $\rho$ uma variável entre - 1 e 1, $n$ o número de amostras aos pares e $d$ a diferença entre as variáveis na Equação 1, o valor positivo de $\rho$ indica correlação direta, já o valor negativo indica correlação inversa e zero a ausência de correlação entre as variáveis (Andriotti, 2003). 0 coeficiente de determinação $\mathrm{R}^{2}$ também foi utilizado para determinar o grau de correlação.

Para associação entre as variáveis foi utilizada a classificação de Spearman, conforme apresentado na Tabela 1.

Tabela 1: Classificação do grau de correlação em função do coeficiente de determinação

\begin{tabular}{ll}
\hline Coeficiente de determinação $\left.\mathbf{( R}^{2}\right)$ & Graus de correlação \\
\hline 0 & Sem Correlação \\
0,01 a 0,19 & Muito Fraco \\
0,20 a 0,39 & Fraco \\
0,40 a 0,59 & Moderado \\
0,60 a 0,79 & Forte \\
0,80 a 0,99 & Muito Forte \\
1 & Perfeito \\
\hline Fonte: Borges (2003) - adaptado pelos Autores. &
\end{tabular}

Os valores da correlação de Spearman indicam que quanto mais próximo do valor 1, em módulo, tem-se uma maior correspondência dos valores correlacionados. Já os coeficientes de determinação de $\mathrm{R}^{2}$, apresentados na Tabela 1 , indicam as correlações, com limite inferior equivalente a 0 para valores sem correlação, e limite superior equivalente a 1 para uma correlação perfeita, indicando que todos os valores das amostras são completamente correspondentes.

\subsection{Correção de BIAS}

Os dados de simulação possuem tendências que precisam ser corrigidas, confrontando um intervalo de tempo base passado com dados observacionais. A metodologia empregada para tanto trata-se de correção de BIAS e obtenção de VIÉS por transformação de Potência, um método não linear de correção de precipitação $(\mathrm{X})$ na forma de $\mathrm{X}^{\mathrm{b}}$, que trata das variâncias temporais (Teutschbein e Seibert, 2012). 0 parâmetro $b$ de correção é obtido através da Equação 2.

$$
f\left(b_{m}\right)=C V_{m}\left(X_{o b s}\right)-C V_{m}\left(X_{\text {sim }}^{b}\right)
$$

Sendo $C V_{m}\left(X_{o b s}\right)$ e $C V_{m}\left(X_{\text {sim }}^{b}\right)$ os coeficientes de variação para cada mês, observado e simulado, obtido pela razão entre o desvio padrão e a média diária pela Equação 3.

$$
C V\left(X_{(d)}\right)=\frac{\sigma\left(X_{(d)}\right)}{\mu\left(X_{(d)}\right)}
$$

Por fim, os dados identificados são então corrigidos pela Equação 4. 


$$
X^{c o r}{ }_{s i m}(d)=X_{\text {sim }}^{b m}(d) \cdot \frac{\mu_{m}\left(X_{(o b s)}\right)}{\mu\left(X^{b m}{ }_{(\operatorname{sim})}\right)}
$$

A função $f\left(b_{m}\right)$ da Equação 2 determina a correção de bias através da diferença entre o coeficiente de variação de dados observados e simulados e esses são obtidos pela razão entre o desvio padrão e a média através da Equação 3. Após esses valores serem obtidos, realiza-se a correção utilizando o modelo presente na Equação 4.

\subsection{Modelo de determinação da quantidade de atrasos/cancelamentos de voos anual com base no clima futuro}

Com base na correlação das variáveis atrasos/cancelamentos de voos e precipitação (P), foram classificados índices de precipitação (Xmm), que representam intervalos baseados na relação desses às operações normais do aeroporto e ao número de atrasos/cancelamentos de voo. Para a situação em que $\mathrm{P}<\mathrm{X}_{1} \mathrm{~mm}$, a operação apresenta-se dentro das normalidades. Já para X1mm $<\mathrm{P}<\mathrm{X}$ mm, o número de atrasos/cancelamentos de voos compreende faixas de $\mathrm{P}$ entre $10 \mathrm{em}$ $10 \mathrm{~mm}$. Com isso, tem-se a quantidade de dias com atrasos/cancelamentos de voos sendo igual ao número de dias de incidência de precipitação no intervalo estabelecido, conforme a Equação 5.

$$
\mathrm{Q}_{\mathrm{atr} / \mathrm{can}}=\mathrm{nd}_{(\mathrm{X} 1 \mathrm{~mm}<\mathrm{P}<\mathrm{Xnmm})}
$$

Na Equação 5 há uma relação entre o número de dias (nd) de atrasos/cancelamentos de voos ao acumulado de precipitação diária simulada para o futuro, em cada intervalo de $10 \mathrm{em} 10 \mathrm{~mm}$ de chuva. Através do nd futuro e passado, pode-se calcular o percentual de aumento dos dias de atrasos/cancelamento de voos analisando o impacto que pode haver devido a esse aumento. Sendo assim, o percentual de aumento de dias de atrasos/cancelamentos de voos resume-se na Equação 6, em que $Q_{\text {fut }}$ refere-se à quantidade de voos atrasados/cancelados no período futuro considerado, e $Q_{\text {pas }}$ refere-se à quantidade de voos atrasados/cancelados no período passado considerado.

$$
\mathrm{P}=\left(\mathrm{Q}_{\text {fut }}-\mathrm{Q}_{\text {pas }}\right) / \mathrm{Qpas}_{\mathrm{p}}
$$

\subsection{Caso de estudo: Aeroporto Internacional de São Paulo/Guarulhos (GRU)}

O Aeroporto Internacional de São Paulo/Guarulhos (GRU), Governador André Franco Montoro, está localizado no município de Guarulhos, estado de São Paulo, situando-se na latitude $23^{\circ} 26^{\prime} 06^{\prime \prime}$ e na longitude 46²8" $22^{\circ}$ W, altitude de 750m, ocupando uma área de aproximadamente $14 \mathrm{~km}^{2}$. GRU apresenta-se como o principal aeroporto internacional do Brasil, operando tanto no transporte de passageiros quanto de cargas. Em 2015 foram registradas cerca de 295 mil movimentações de aeronaves e um total de cerca de 39 milhões de passageiros, sendo que o aeroporto detém próximo de $20 \%$ de toda movimentação de passageiros na aviação regular do país (GRU AIRPORT, 2016). Isso faz de GRU um significativo exemplar a ser utilizado como referência nesse estudo. 


\section{ANÁLISE DOS RESULTADOS}

\subsection{Correlação entre atrasos/cancelamentos de voos por evento de chuva}

Através de uma série histórica de números de voos em cinco anos (2011-2015), foi classificada a ocorrência de atrasos e cancelamentos de voos por motivos diversos, passando para a incidência por eventos meteorológicos e, por fim, por incidência de chuvas extremas, conforme descrito na Tabela 2.

Tabela 2: Dados de atrasos e cancelamentos de voos (2011-2015)

\begin{tabular}{lll}
\hline Número de voos chegada e partida & Atrasos por chuvas intensas & Cancelamentos por chuvas extremas \\
\hline 1427700 & 1285 & 145 \\
\hline
\end{tabular}

Com os dados de chuvas obtidos pelo SIGRH (2015) para a área de estudo, obteve-se os seguintes resultados classificados por acumulado de precipitação diária em cinco anos (20112015), e pode-se observar uma relação direta dos altos valores acumulados de chuva com possíveis atrasos e cancelamentos de voos. Foi identificada a correlação de 0,91 que, conforme Spearman, apresenta-se como uma correlação muito forte para a maioria dos dias analisados dentro de uma faixa de valores acumulados, conforme Figura 1, que apresenta a análise de 48 eventos (dias) considerados no estudo.

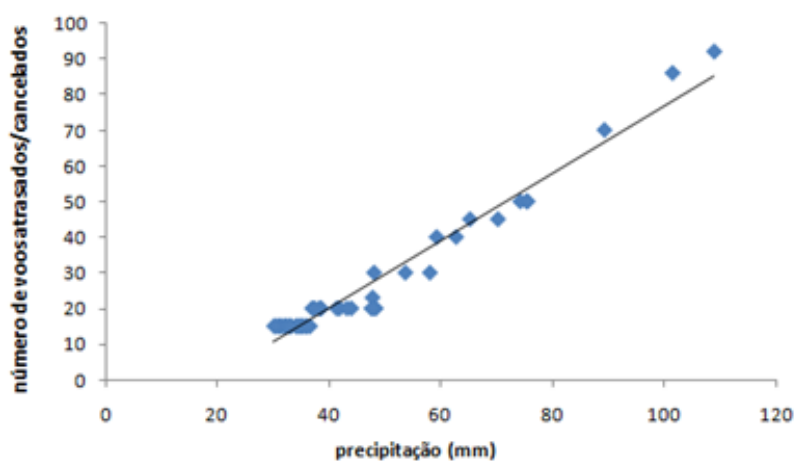

Figura 1. Correlação de Spearman para chuva $x$ atrasos e cancelamentos de voos

De acordo com os valores dos 48 eventos (dias) analisados e apresentados na Figura 1, dos quais há valores muito próximos que foram agrupados, foi identificado um intervalo de precipitação correlacionado aos atrasos/cancelamentos de voos, conforme Tabela 3.

Tabela 3: Intervalo pluviométrico correlacionado (Fonte: Dados da pesquisa)

\begin{tabular}{ll}
\hline Intervalo pluviométrico & Relação atrasos/cancelamentos de voos (valor médio) \\
\hline Chuva $<30 \mathrm{~mm}$ & Operação normal \\
$30 \mathrm{~mm} \leq$ chuva $<40 \mathrm{~mm}$ & 15 \\
$40 \mathrm{~mm} \leq$ chuva $<50 \mathrm{~mm}$ & 20 \\
$50 \mathrm{~mm} \leq$ chuva $<60 \mathrm{~mm}$ & 30 \\
$60 \mathrm{~mm} \leq$ chuva $<70 \mathrm{~mm}$ & 40 \\
$70 \mathrm{~mm} \leq$ chuva $<80 \mathrm{~mm}$ & 45 \\
$80 \mathrm{~mm} \leq$ chuva $<90 \mathrm{~mm}$ & 60 \\
$90 \mathrm{~mm} \leq$ chuva $<10 \mathrm{~mm}$ & 70 \\
$100 \mathrm{~mm} \leq$ chuva $<110 \mathrm{~mm}$ & 85 \\
$110 \mathrm{~mm} \leq$ chuva $<120 \mathrm{~mm}$ & 105 \\
\hline
\end{tabular}


De acordo com a Tabela 3, para chuvas abaixo de 30mm adotou-se ausência de atrasos ou cancelamentos de voos devidos ao histórico analisado, em que foi classificado como dias de operações normais no aeroporto. Já para o intervalo entre 30 e $40 \mathrm{~mm}$ houve aproximadamente 15 voos com incidência de atrasos ou cancelamentos, e assim por diante até dias com mais de $110 \mathrm{~mm}$ de precipitação acumulada ultrapassarem 100 casos de atrasos ou cancelamentos de voos.

\subsection{Correção dos dados de precipitação simulada}

A partir dos dados observacionais e os dados de base de 2011-2015 utilizados na simulação futura, foram realizadas as correções através do método de transformação de potência, para a determinação do coeficiente exponencial $b$ mensal, conforme Tabela 4 . Utilizando-se desses valores, aplicou-se a correção para o futuro através de uma série temporal de 2016 a 2020. Como os valores corrigidos se baseiam nos valores observados, a correlação também se apresenta adequada para a utilização dos novos dados.

Tabela 4: Coeficientes de correção $b$

\begin{tabular}{ll}
\hline Mês & $\boldsymbol{b}$ \\
\hline Janeiro & 1,67 \\
Fevereiro & 1,65 \\
Março & 1,70 \\
Abril & 1,64 \\
Maio & 1,52 \\
Junho & 1,51 \\
Julho & 1,58 \\
Agosto & 1,51 \\
Setembro & 1,77 \\
Outubro & 1,74 \\
Novembro & 1,81 \\
Dezembro & 1,68 \\
\hline
\end{tabular}

Cada valor presente na Tabela 4 representa a potência $b$ da Equação 2 calculada mensalmente para se obter a correção dos BIAS e, consequentemente, corrigir os dados brutos simulados afim de se obter os novos dados para a aplicação da Equação 5 e, assim, identificar o aumento dos dias de atrasos/cancelamentos de voos futuros.

\subsection{Aumento percentual de atrasos/cancelamentos de voos futuros}

A partir das simulações futuras corrigidas, foram obtidos os dias com precipitações nos intervalos pré-estabelecidos e confrontados com o intervalo pluviométrico correlacionado. $\mathrm{O}$ aumento do número de dias com precipitação geradora de atrasos/cancelamento de voos e a nova quantidade de dias de atrasos/cancelamentos de voos foram estabelecidos, além da porcentagem do aumento dessas variáveis, conforme apresentado nas Equações 5 e 6. Os valores correspondentes são apresentados na Tabela 5.

Como se pode-se perceber pela Tabela 5, de acordo com os cálculos e simulações realizadas, haverá um aumento significativo em relação ao número de atrasos/cancelamentos de voo devido aos acúmulos de precipitações, com algo em torno de 186 atrasos/cancelamentos adicionais em 5 anos, um aumento de 13,9\%, passando de 1330 na base considerada para 1516 nos próximos 5 anos considerados. Esse aumento também acarretará em um aumento de 10 dias 
críticos paras as operações de pousos e decolagens em GRU, nos quais estima-se que não haverá operações normais no aeroporto, contribuindo para prejuízos e transtornos de considerável escala ao aeroporto em função da sua significativa movimentação.

Tabela 5: Comparação dos dados de base e futuro

\begin{tabular}{cccc}
\hline $\begin{array}{l}\text { Atrasos/cancelamentos de voos } \\
(\mathbf{2 0 1 1 - 2 0 1 5 )}\end{array}$ & $\begin{array}{l}\text { Atrasos/cancelamentos de voos } \\
(\mathbf{2 0 1 6 - 2 0 2 0 )}\end{array}$ & Percentual de aumento & $\begin{array}{l}\text { Aumento do número } \\
\text { de dias }\end{array}$ \\
\hline 1330 & 1516 & 13,9 & 10 \\
\hline
\end{tabular}

Nota-se que GRU possui suas operações suportadas por ILS (Instrument Landing System), ou seja, se nesse aeroporto foi identificado esse aumento e comprometimento das operações, em aeroportos desprovidos dessas tecnologias certamente os impactos seriam ainda mais significativos. Estudos futuros relacionados ao uso dessas tecnologias ou aos custos dos cancelamentos/atrasos, por exemplo, poderão mesurar o impacto de tais aumentos nas operações aeroportuárias.

\section{CONSIDERAÇÕES FINAIS}

Apesar de haver trabalhos descrevendo a relação entre chuvas e atrasos/cancelamentos de voos, foram identificadas carências de estudos que correlacionem as variáveis aqui consideradas e sua relação às situações climáticas e simulações para o clima futuro. As contribuições desse estudo também estão relacionadas à proposição de um modelo a partir da análise de índices, para cada faixa de intensidade de chuva, relacionados à quantidade de atrasos/cancelamentos de voos para eventos futuros.

0 estudo realizado comprovou a forte correlação entre atrasos/cancelamentos de voos por evento de precipitação, com um intervalo de acumulados de precipitação. Após a correção dos dados simulados obteve-se um aumento do número de dias com precipitação pertencente aos intervalos de acumulados de chuva e, consequentemente, um aumento do número de atrasos/cancelamentos de voos próximo a 14\%, acarretando em 186 novos voos que terão alterações, bem como um acréscimo de 10 dias em que haverá alterações nas operações do aeroporto.

Estudos futuros podem ser desenvolvidos no sentido de mensurar esses atrasos/cancelamentos de voos em diferentes intervalos de horários e confrontar com as possíveis alterações climáticas pluviométricas, ou ainda correlacionar atrasos e cancelamentos de voos de modo distinto às precipitações. Outra sugestão seria aplicar essa metodologia a outros aeroportos, principalmente em operações VFR, sem uso de IFR como aqui analisado no caso de GRU, o que tornaria o estudo ainda mais relevante do ponto de vista das operações aeroportuárias. Considerar também o aumento das operações do aeroporto poderia ser outra possibilidade de análise para futuros estudos.

\section{AGRADECIMENTOS}

Conselho Nacional de Desenvolvimento Científico e Tecnológico - CNPq (Processo: 160348/2015-3, PDJ), Fundação de Amparo à Pesquisa do Estado de Goiás (FAPEG) e aos revisores, pelas excelentes contribuições para a melhoria do estudo.

\section{REFERÊNCIAS}

Alves, T. L. B.; J. V. V. Azevedo; C. A. C Santos e P. V. Azevedo (2015) Influence of climatic variations in the occurrence of diseases of the upper airways in the municipality of Monteiro-PB. Ciência e Natura. v. 37, n.4, p. 433-450. DOI: 105902/2179460X16702.

Andriotti, J. L. S. (2003) Fundamentos de Estatística e Geoestatística. Ed. UNISINOS, São Leopoldo. 
Bendinelli, W. E.; H. F. A. J. Bettini e A. V. M. Oliveira (2016) Airline delays, congestion internalization and non-price spillover effects of low cost carrier entry. Transportation Research. Part A, Policy and Practice, v. 85, p. 39-52, DOI: 10.1016/j.tra.2016.01.001

Benedetto, A. (2002) A decision support system for the safety of airport runways: the case of heavy. Transportation Research Part A: Policy and Practice, v. 36, n. 8, p. 665-68. DOI: 10.1016/S0965-8564(01)00029-5.

Borges, B. L. M. (2003) Simplificando a estatística. Campina Grande: EDUEPB, 2008p.

GRU Airport, Aeroporto Internacional de São Paulo/Guarulhos. Disponível em: http://www.gru.com.br (Acesso em: 19/05/2017)

Halkos, G. E. e K. D. Tsilika (2014) Analyzing and visualizing the synergistic impact mechanisms of climate change related costs. Applied Mathematics and Computation. v. 246, p. 586-596. DOI: 10.1016/j.amc.2014.08.044.

Koetse, M. J. e P. Rietveld (2009) The impact of climate change and weather on transport: An overview of empirical findings. Transportation Research Part D: Transport and Environment. v. 14, n. 3, p. 205-221. DOI: 10.1016/j.trd.2008.12.004.

Janić, M. (2015) Modelling the resilience, friability and costs of an air transport network affected by a large-scale disruptive event. Transportation Research Part A: Policy and Practice. v. 71, p. 1-16. 2015. DOI: 10.1016/j.tra.2014.10.023.

Metchko, P. A e M. A. Monteiro (2014) Windshear: A condição meteorológica de risco para a aviação. IX EPCT - Encontro de Produção Científica e Tecnológica - UNESPAR. Campo Mourão.

Santos, T. A. e G Fisch. (2016) Temperatura e precipitação: futuros cenários do município de Taubaté, SP, Brasil. Revista AmbiAgua. Taubaté. v. 11, p. 1068-1087. D0I:10.4136/ambi-agua.1896.

Santos, G. e M. Robin (2010) Determinants of delays at European airports. Transportation Research Part B: Methodological.v. 44, n. 3, p. 392-403. DOI: 10.1016/j.trb.2009.10.007.

SIGRH - Sistema Integrado de Recursos Hídricos do estado de São Paulo (s/d). Disponível em: http://www.sigrh.sp.gov.br (Acesso em: 05/03/17).

Silva, J. P. S. (2008) Aderência pneu-pavimento em revestimentos asfálticos aeroportuários. Dissertação de Mestrado em Geotecnia. Programa de Pós-graduação em Geotecnia. Universidade de Brasília. 134p.

Teutschbein, C. e J. Seibert (2012) Bias correction of regional climate model simulations for hydrological climate-change impact studies: review and evaluation of different methods. Journal of Hydrology. v. 12, p. 456-457. DOI: 10.1016/j.jhydrol.2012.05.052

Xiong, J. e M. Hansen (2013) Modelling airline flight cancellation decisions. Transportation Research Part E: Logistics and Transportation Review. v. 56, p. 64-80. DOI: 10.1016/j.tre.2013.05.003.

Walters, K. M. e M. B. Sebens (2016) Using climate change scenarios to evaluate future effectiveness of potential wetlands in mitigating high flows in a Midwestern U.S.watershed. Ecological Engineering. v. 89, p. 80-102. DOI: 10.1016/j.ecoleng.2016.01.014. 